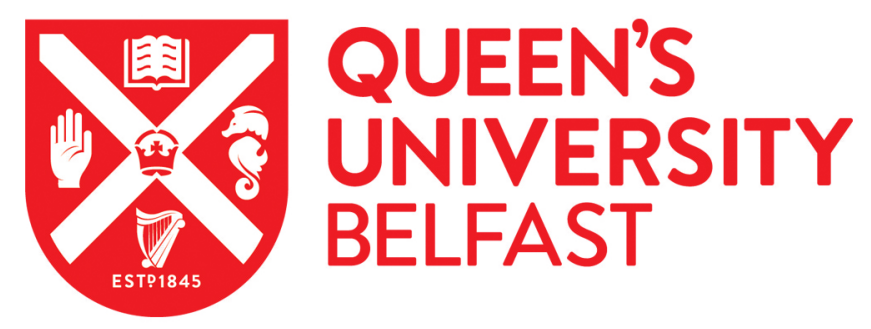

\title{
Active Surveillance for Favourable-Risk Prostate Cancer: Is there a Greater Psychological Impact than Previously Thought? A Systematic, Mixed Studies Literature Review.
}

Ruane-McAteer, E., Porter, S., O'Sullivan, J. M., Santin, O., \& Prue, G. (2016). Active Surveillance for Favourable-Risk Prostate Cancer: Is there a Greater Psychological Impact than Previously Thought? A Systematic, Mixed Studies Literature Review. Psycho-oncology. https://doi.org/10.1002/pon.4311

Published in:

Psycho-oncology

\section{Document Version:}

Peer reviewed version

Queen's University Belfast - Research Portal:

Link to publication record in Queen's University Belfast Research Portal

\begin{abstract}
Publisher rights
Copyright 2016 Psycho-oncology

This is the peer reviewed version of the following article: Ruane-McAteer, E, Porter, S, O'Sullivan, JM, Santin, O \& Prue, G 2016, 'Active Surveillance for Favourable-Risk Prostate Cancer: Is there a Greater Psychological Impact than Previously Thought? A Systematic, Mixed Studies Literature Review.' Psycho-Oncology, which has been published in final form at

http://onlinelibrary. wiley.com/wol1/doi/10.1002/pon.4311/abstract. This article may be used for non-commercial purposes in accordance with Wiley Terms and Conditions for Self-Archiving.

General rights

Copyright for the publications made accessible via the Queen's University Belfast Research Portal is retained by the author(s) and / or other copyright owners and it is a condition of accessing these publications that users recognise and abide by the legal requirements associated with these rights.
\end{abstract}

Take down policy

The Research Portal is Queen's institutional repository that provides access to Queen's research output. Every effort has been made to ensure that content in the Research Portal does not infringe any person's rights, or applicable UK laws. If you discover content in the Research Portal that you believe breaches copyright or violates any law, please contact openaccess@qub.ac.uk. 
Manuscript title: Active Surveillance for Favourable-Risk Prostate Cancer: Is there a

Greater Psychological Impact than Previously Thought? A Systematic, Mixed Studies

Literature Review.

Running Head: The Psychological Impact of Active Surveillance for Prostate Cancer

1. *Eimear Ruane-McAteer ${ }^{\mathrm{a}}$

eruanemcateer01@qub.ac.uk $+44(0) 2890972684$

*Corresponding author

2. Prof Sam Porter, $\mathrm{PhD}^{\mathrm{a}}$

3. Prof Joe M. O’Sullivan, MD ${ }^{\mathrm{b}, \mathrm{c}}$

4. Dr Olinda Santin, $\mathrm{PhD}^{\mathrm{a}}$

5. Dr Gillian Prue, $\mathrm{PhD}^{\mathrm{a}}$

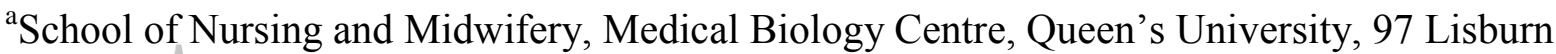

Road, Belfast BT9 7BL

${ }^{\mathrm{b}}$ The Northern Ireland Cancer Centre (NICC), Belfast City Hospital, 51 Lisburn Road,

Belfast BT9 7AB

${ }^{\mathrm{c}}$ Centre for Cancer Research and Cell Biology (CCRCB), Queen's University Belfast, 97

Lisburn Road, Belfast BT9 7AE

Funding: This review was completed for part-fulfilment of ER's PhD. No outside funding

was used.

Conflict of interest: The authors declare no conflicts of interest.

\section{Author contribution}

ER and GP were responsible for the design of this systematic review, data acquisition, analysis and interpretation. OS aided in this process, arbitrating any disagreement. SP and JO'S advised on design and evidence acquisition. ER drafted the article. GP, OS, SP and JO'S critically revised the manuscript. All authors approved the final submission.

This article has been accepted for publication and undergone full peer review but has not been through the copyediting, typesetting, pagination and proofreading process which may lead to differences between this version and the Version of Record. Please cite this article as doi: $10.1002 /$ pon.4311 


\begin{abstract}
Objective

Active Surveillance (AS) allows men with favourable-risk prostate cancer (PCa) to avoid or postpone active treatment and hence spares potential adverse side effects for a significant proportion of these patients. Active surveillance may create an additional emotional burden for these patients.
\end{abstract}

The aim of the review was to determine the psychological impact of AS to inform future study in this area and to provide recommendations for clinical practice.

Methods

Studies were identified through database searching from inception to September 2015.

Quantitative or qualitative non-interventional studies published in English that assessed the psychological impact of AS were included. The Mixed Methods Appraisal Tool was used to assess methodological quality.

Results

Twenty-three papers were included (20 quantitative, 3 qualitative). Quantitatively, the majority of patients do not report psychological difficulties, however when appropriateness of study design is considered, the conclusion that AS has minimal impact on wellbeing, may not be accurate. This is due to small sample sizes, inappropriately timed baseline, and inappropriate/lack of comparison groups. In addition, a mismatch in outcome was noted between the outcome of quantitative and qualitative studies in uncertainty, with qualitative studies indicating a greater psychological impact.

\title{
Conclusions
}

Due to methodological concerns, many quantitative studies may not provide a true account of the burden of AS. Further mixed-methods studies are necessary to address the limitations 
highlighted and to provide clarity on the impact of AS. Practitioners should be aware that despite findings of previous reviews, patients may require additional emotional support.

\section{Keywords}

Prostatic Neoplasms. Cancer. Oncology. Active Surveillance. Anxiety. Depression.

Uncertainty.

\section{Background}

Active surveillance (AS) was developed in response to the increasing prevalence of lower risk prostate cancer (PCa) in older men [1]. AS allows the majority of patients with lower risk PCa to avoid active treatment and hence the side-effects associated with such therapy [2]. Although AS protocols may vary, they usually involve Prostate-Specific Antigen (PSA) tests and Digital Rectal Examinations (DRE) at regular intervals, and annual/biannual biopsies. In recent years, regular multi-parametric MRI scans of the prostate have become part of AS protocols [3]. Numerous studies have documented the appropriateness of AS from a medical perspective, however the psychological impact of AS remains understudied [4]. In spite of this, the assumption is that men undergoing AS do not require additional support, psychological or otherwise, throughout this monitoring period. This assumption can partly be attributed to results of systematic reviews in this area, for example: a recent exclusively quantitative systematic review [5] concluded that men on AS reported no major difficulties in quality of life (QoL) or psychological wellbeing. However, this review utilised narrow inclusion and exclusion criteria and excluded studies referring to Watchful Waiting (WW), a management approach that is palliative in nature but often incorrectly used interchangeably with AS. This omission may have led to the exclusion of some relevant papers. The lack of critical appraisal meant study quality was not taken into consideration in the interpretation of the results. 
In a similar quantitative review [6], it was concluded that AS was unlikely to be associated with an adverse effect on general psychological wellbeing. In this instance, studies describing WW or 'no treatment' were included. However, no distinction was made between those studies describing AS versus true WW. Although the methodological quality of studies was assessed, this was not considered when interpreting the results of the review. In addition, neither of these reviews included qualitative studies, which meant that an important opportunity to better understand the experiences of patients was missed. It is our contention that a mixed methods review that includes both qualitative and quantitative studies would allow for richer experiential data to be included without compromising generalisability achieved using quantitative methods [7].

Aims

The aim of this systematic mixed studies review was to synthesise and appraise the quantitative and qualitative knowledge to develop a more comprehensive picture of published studies reporting the psychological impact of undergoing AS.

\section{Evidence acquisition}

The Preferred Reporting Items for Systematic Reviews and Meta-Analyses (PRISMA) guidelines were used for the conduct and reporting of this systematic review [8].

Eligibility criteria

Inclusion criteria: non-interventional studies published in English assessing the psychological impact of AS in lower-risk PCa, including studies comparing AS with AT. Studies referring to WW were included when the definition was that of AS.

Exclusion criteria: review articles, editorials, comments, intervention studies (e.g. studies that included a psychosocial intervention), needs assessments and studies assessing quality of life (QoL). While QoL is an important factor, it was deemed inappropriate for the present review 
due to the insensitivity of general QoL measures in assessing clinical change in psychological functioning [9].

\section{Information sources}

Medical and nursing databases were searched from inception between August and September 2015 with no limitations on time, using a predetermined search strategy (Fig.1.). Titles and abstracts were screened by ER and GP based on the inclusion/exclusion criteria, where there was doubt regarding the eligibility of a particular title or abstract the record was retained for full-text screening. OS arbitrated any disagreement at the full-text screening stage.

Search

Figure 1. PRISMA Flow Diagram

\section{Study selection}

Qualitative and quantitative findings related to the prevalence and predictive/protective factors of psychological variables were reported, namely depression, anxiety, and uncertainty. ER and GP extracted data from each article and applied the quality appraisal tool, OS arbitrated any disagreement.

\section{Data collection process}

The Mixed Methods Appraisal Tool (MMAT) [10], an appropriate, reliable and efficient tool for mixed studies reviews was used to assess study quality [11]. ER and GP completed the MMAT for each study, with OS arbitrating. This appraisal directly informed the interpretation of study findings.

\section{Results}

Synthesis of results

Twenty-three papers were included, 20 quantitative [12-31], and 3 qualitative [32-33]. Nine were longitudinal [15, 18-21, 23, 25-27] with a follow-up period ranging from 9 months [25] to 3 years [18]. Attrition in these studies varied, with response rates at follow-up time-points 
ranging from $13 \%$ [21] to $89 \%$ [15]. Three studies did not report attrition [18, 20, 23]. Four of the 9 longitudinal studies had follow-up response rates of $>70 \%[15,19,25,27]$, whereas others reported more conservative follow-up response rates of $<60 \%[21,26]$. Fourteen were cross-sectional [12-14, 16-17, 22, 24, 28-34], with time since diagnosis ranging from 2 months [23-24, 29] to 136 months [22]. Six papers referring to WW, yet providing definitions for AS, i.e. not palliative care, were included [17-18, 23, 32]. Seven papers included comparison groups; the majority compared AS patients with patients opting for AT [14, 15, 18, 21, 23, 26], one was a comparison of North American and Irish AS patients [17]. Breakdown of individual sample sizes and countries of origin are detailed in Table 1. The pooled number of AS patients across the twenty-three papers amounted to 1777 men.

\section{Quality appraisal}

Using the MMAT [11], papers were scored against four main criteria associated with the specific research design; four papers met $100 \%$ of criteria; 15 met $50-75 \%$; and four met only $25 \%$ of the methodological quality criteria (Table 1). Failure to justify sample size, inappropriate/no comparison group, and lack of baseline measures were the most frequently observed methodological issues.

\section{Depression}

Twelve quantitative studies investigated depression in this population, reporting data for 1007 AS patients in total. Five studies included AT men as a comparison group [14, 15, 18, 23, 26]. Six different scales were used to assess depression (Table 2).

\section{Quality appraisal}

Eight of the 12 studies were considered high quality studies (75\%-100\% of methodological criteria was met). Two of the studies fulfilled $50 \%$ of the methodological criteria $[15,22]$; with an insufficient response rate, failure to provide reasons for non-participation and an inability to determine if the sample was representative due to the authors failing to report 
demographic information per treatment group included were limitations of these studies. Two studies met only $25 \%$ of the methodological quality criteria [16, 29]. The first [16] failed to justify sample size, provide reasons for non-participation and recruited from support groups, leading to a potential selection bias. Response rate was also not reported [16]. In the second [29], the HADS was used inappropriately to diagnose clinical depression. HADS does not include somatic symptoms that makes up the diagnostic criteria of clinical depression therefore the measure used does not address the aims of their study. Other methodological issues included lack of a representative sample, as despite the use of multiple sites $95 \%$ of the sample identified as white British. The authors' failure to include a control group also reduced the MMAT score attributed to the study. The study did however include a large number of participants $(\mathrm{n}=313)$ and their response rate was high $(73.47 \%)$.

\section{Prevalence}

Four studies reported prevalence data for depression [14, 21, 25, 29]. Generally, the prevalence of depression was low; two studies reported mild depression ranging from 4-11\% $[14,21]$, with moderate-severe depression reported in less than $5 \%$ of both AS and RP patients [21]. One study reported clinically significant depression in $12.5 \%$ of their sample [29]. There was disagreement regarding the severity of depression in comparison to noncancer men. One study reported that although mild, depression was higher than literature reporting depression scores for men without a PCa diagnosis [14]. Conversely, depression levels were reportedly similar to normative data of clinical populations [25]. However, the latter study utilised a prospective, longitudinal design, with low attrition rates therefore reducing the impact of individual differences and increasing credibility of the findings. When compared to curative treatment, AS patients had the most favourable depression score $[15,26]$. Although the difference between AS and RP patients immediately post- 
diagnosis/early treatment was statistically significant, clinical significance was not reported $[15]$.

One low quality study [29] reported a higher number of participants scoring within clinical levels; although the mean score of depression was low, $12.5 \%$ of patients' scores suggested presence of clinical depression.

\section{Factors associated with depression}

Five studies attempted to identify factors predicting depression (Table 3). Neurotic patients who experienced a major life event additional to their diagnosis demonstrated increased depression [24]. Extraversion, continued sexual activity, and higher QoL were associated with decreased depression [24]. Similarly, patients with higher QoL and low neuroticism reported lower depression at diagnosis [25]. It was concluded that patient-bound factors, e.g. personality, were the most important determinants of depressive symptoms [13, 15, 24]. Lack of a partner and impaired mental health ( $\mathrm{MH})$ were both predictive of poorer wellbeing [13]. Patients enrolled in AS protocols soon after diagnosis were more likely to adopt poor coping strategies and demonstrate maladaptive adjustment to cancer; these patients had less time to seek information to support their choice of AS and therefore understand that their PCa was manageable [13].

Change in depression over time

Six studies assessed depression longitudinally [15, 21, 23, 25-27]. Depression was reported to decrease with time in five studies $[15,21,23,26-27]$ for both mild $[15,21]$ and clinically significant depression $[15,29]$. Conversely, in another high quality study with one of the lowest attrition of all included studies, depression remained stable up to 18-months postdiagnosis [25]. A high quality cross-sectional study [14] reported a correlation between increased time since diagnosis and increased depression; a finding that must be treated with caution given the limitations of cross-sectional designs. 


\section{Anxiety}

Eighteen quantitative studies measured anxiety in 1639 AS patients [12-15, 18-30], using eight different scales (Table 2). Six studies included comparison groups of AT men [14, 15, $18,21,23,26]$.

\section{Quality appraisal}

Eleven of the 18 studies assessing anxiety met $75 \%$ or $100 \%$ of the methodological quality criteria set out in the MMAT. Four met $50 \%$ of the criteria $[12,15,18,22]$, issues included a failure to report reasons for the low response rate $[12,18,22]$, potential selection bias and failure to report the demographic information divided by treatment group [15], and no standardised tool for assessment of anxiety [18]. Three studies met $25 \%$ of the methodological quality criteria $[16,28,29]$, issues with these studies included low sample size, potential selection bias, insufficient response rate or failure to report the response rate, reasons for non-participation not explained, failure to include a control group, and inappropriately timed baseline measurements.

\section{Prevalence}

The prevalence of anxiety ranged from $13-45 \%[12,14,21-22,24-25,27,29-30]$. One study reported up to $5 \%$ of patients with moderate-severe anxiety levels [21], and one with almost $25 \%$ of participants with clinical levels of anxiety [29]. The majority of studies reported anxiety comparable or lower than data from non-clinical populations $[12,14,22,24]$. One study [21] reported that the majority of participants had anxiety higher than that of noncancer men; however, the study cited to support these claims included no non-cancer data [35].

AS men appeared to have low anxiety when compared to patients opting for AT [15]. Only one study directly contradicted these findings [26]; however this can be attributed to potential 
selection bias in the increased psychological dysfunction reported by those completing follow-up.

\section{Change in anxiety over time}

Six studies examined the temporal variability in anxiety [19-20, 21, 25-27]. With the exception of one study [25], statistically significant declines were observed over time. However, the one contradictory study [25] was of high methodological quality and maintained a high response rate during study follow-up. One study reported that although $20 \%$ of patients suffered from clinically significant anxiety levels at baseline (within 6 months of diagnosis), only $5 \%$ of the total sample chose to leave the AS protocol due to the psychological burden [27]. A significant decrease was observed in general anxiety and fear of disease progression over the course of the 18 month follow-up [27]. A number of other studies support these findings, also concluding that anxiety remained either stable or reduced over time $[15,20,23-24,26]$.

In a 30-month follow-up study [19], anxiety reduced significantly at 18- and 30-months postdiagnosis. Interestingly, 12- and 24-month follow-up data were not significant; typically this is when patients receive a biopsy to reassess their cancer and resulting course of treatment. Although, it must be noted that the trend of declining anxiety remained consistent across these time points.

Longitudinal studies documenting a general decline in anxiety during AS are supported by two of the three cross-sectional studies that asked men to report number of months spent on AS [14, 21, 24]. Although not significant, increased time undergoing AS was associated with stable or decreased anxiety $[22,24]$. One cross-sectional study however found a significant increase in anxiety with reported increased time since diagnosis [14].

Individual differences are an important factor and cross-sectional studies must be interpreted with caution despite apparent high methodological quality, this study design may simply not 
be appropriate to capture these men's experiences. Based on the results of the previously reported, high quality study [19](Table 1), anxiety appeared to fluctuate, therefore, the time point at which the cross-sectional studies assess men would be a crucial in terms of the anxiety reported, and may explain some of the conflicting results discussed.

Factors associated with anxiety

The factors that appeared to be predictive of anxiety were: high neuroticism [24], younger age at diagnosis [14], low QoL and fear of cancer recurrence [12], impaired $\mathrm{MH}$, lack of a partner and decreased number of cores taken at diagnostic biopsy [13], patients' relationship status i.e. single/divorced $[13,29]$, misunderstanding of AS and resulting deferral of decision making to physician $[13,25,30]$ (Table 3 ). The finding in relation to decreased number of cores taken at diagnostic biopsy, the authors suggested that this may a result of the patients' perception, however inaccurate, that more of their cancer had been removed [13]. High neuroticism and high PSA were associated with increased PCa-specific anxiety [24]. Fear of disease progression, a component of $\mathrm{PCa}$-specific anxiety, was identified as a trigger for discontinuation of AS in favour of AT [19]. The combination of high QoL and low neuroticism, was reported to be significant in minimising anxiety [25].

\section{Uncertainty}

Six studies assessed uncertainty in 266 AS men. Three studies were qualitative [32-34], and three quantitative $[17,19,28]$. The quantitative studies used MUIS (Table 2) to measure uncertainty. Four additional papers were included in the uncertainty theme due to their assessment of decisional conflict [16, 24, 25, 27], a state of uncertainty, using the DCS (Table

2). One study included a comparison group of Irish and North American AS patients [17].

\section{Quality Appraisal}

Of the ten studies assessing uncertainty in the AS population, only one met $100 \%$ of the methodological quality criteria in the MMAT [24]. Three studies met $75 \%$ of the criteria [19, 
$25,27]$, the absence of a comparison group in two of these studies prevented them from meeting all the methodological quality criteria [25, 27]; whereas potential selection bias in the recruitment of participants was an issue in the third study [19]. One qualitative study [32] also met $75 \%$ of the methodological criteria, as a result of failing to acknowledge their influence on their data. The two remaining qualitative studies $[33,34]$ met $50 \%$ of the methodological quality criteria due to the lack of acknowledgement of the researchers influence and the impact the context within which the research took place had upon the participants and resulting data. Two quantitative studies also met just $25 \%$ of the methodological criteria [16, 28]; failure to discuss the justification for their specific sample size, reasons for potential participants' non-participation, response rate and issues with sampling were the reasons for this. Finally, one additional paper met only $25 \%$ of the methodological criteria [17], this was due to the small sample ( $n=29$ participants), failure to apply appropriate inferential statistics as a result, as well as a failure to report the response rate.

\section{Prevalence}

None of the included studies reported the prevalence of clinically significant uncertainty using the MUIS. However, approximately $25 \%$ of patients scored clinical levels in DCS [24$25,27]$.

The perception of uncertainty

The three qualitative studies included in this review identified similar themes regarding the nature of uncertainty experienced during AS, which contrasted with the quantitative findings. Men described 'intolerable uncertainty', a 'Dangerous Wait', characterised by uncertainty and a perception of 'risking one's life' [33]. Although this study included only those who had converted to AT which may overstate the impact of uncertainty in AS. The other qualitative studies interviewed men who remained on AS $[32,34]$. The theme 'To be Uncertain, Afraid, 
Worried' emerged in one of these qualitative studies [32] in participants still undergoing AS with respondents describing constant threat, fear and worry.

While patients understood the information about their diagnosis and prognosis intellectually, they had not integrated the message emotionally [32]. An overarching theme of uncertainty prevailed in each participant's account, either implicitly or explicitly [32]. This theme was characterised similarly to those previously reported: persistent uncertainty surrounding mortality and potential spreading, potential need for AT, and patients' ability to cope with treatment-induced morbidities. Participants described living in 'shadowland' while they 'waited for a disaster' [232]. These subthemes were related back to patients' masculine identities: pressure to maintain sexual function, and to continue to provide financial stability for their families [34]. Qualitatively, participants described uncertainty as featuring more significantly throughout the AS experience than the quantitative data suggested, a finding warranting further study.

\section{Change in uncertainty over time}

Three of the included uncertainty studies assessed uncertainty longitudinally [19, 25, 27].

Attrition was generally low at initial follow-up points, with response rates $>70 \%[19,25,27]$, however after 18 month follow-up response rate dropped to 67\% [27] and $44 \%$ after 2 year follow-up [19]. Uncertainty decreased from baseline to 18-months, however this decrease was neither statistically nor clinically significant [27]. Uncertainty at 6-months post-diagnosis predicted scores after 9-months of AS, suggesting uncertainty remains stable within the first year. A significant decrease was found from baseline up to 30-months post-diagnosis [19] however attrition may have been an issue. Conversely, qualitatively patients reported that uncertainty was time sensitive and peaked leading up to monitoring appointments, PSA and biopsy results [34]. 
A number of factors were reported to increase uncertainty, including high neuroticism and increased role of clinician in decision-making $[24,25]$. Patients who experienced depression, and had a more negative outlook were less satisfied with their treatment decision [16] (Table

3). Uncertainty was reported to be a significant factor, and had a resulting impact on QoL and fear of disease progression $[19,28]$. It was reported that it was the perception of danger associated with AS that increased uncertainty and had the resultant impact on QoL [28].

Anxiety was also associated with uncertainty [28].

Factors reported to decrease uncertainty and decisional conflict were also discussed in the literature: high extraversion and the management of $\mathrm{PCa}$ in a university/specialist hospital appeared to be associated with lower decisional conflict $[24,25]$. Palpable disease and older age at diagnosis reportedly had an additional favourable effect on the perceived risk of progression, a form of uncertainty, at follow up [25]. The finding in relation to palpable disease appears to be counter-intuitive, the authors posited that older patients with palpable disease at diagnosis may experience higher uncertainty initially, yet following a period of time on surveillance show greater improvement upon realising the feasibility of surveillance [25]. Favourable MH, optimism and higher self-efficacy (SE) as well as perceived consistency in medical information was associated with reduced uncertainty [16]. Qualitative data suggested stable or decreased disease characteristics at follow-up reduced uncertainty surrounding impending follow-up appointments and delays between monitoring appointments and receipt of results [34]. Patients also discussed feeling more secure when they saw the same clinician at follow-up appointments [34].

The role of clinicians was ambiguous. They were both sources of uncertainty in that they were potentially bearers of bad news that the cancer had progressed further, and of security in that they provided patients with the reassurance of regular check-ups [32]. 
Patients appeared to cope with uncertainty and decisional conflict in various ways, as was described in two qualitative studies [32, 34]. With regard to decision making, patients appeared to assume either a passive or active role in the process; while some patients opted to defer decision-making power entirely to their clinician, others chose to actively seek out further information or request a second opinion to engage more with the decision-making process [32]. Similarly, in response to diagnosis, some patients reported they decided to 'screen off' their cancer by setting aside feelings of threat or completely denying the existence of their cancer, while others compensated for the perceived threat of their cancer via lifestyle change [32]. Patients also described control as central to their coping, this control was asserted by 'living a normal life' [34], similar to 'screening off' [32], or by 'doing something extra', a theme that also converges with lifestyle change discussed previously [32].

\section{Discussion}

Main Findings

From a quantitative perspective it would appear that men undergoing AS show favourable psychological wellbeing, with only a small number reporting maladjustment. These findings are consistent with those of previous systematic reviews $[5,6]$ in spite of differing inclusion criteria particularly in relation to inclusion of papers referring to AS as WW in the present review. While this convergence strengthens confidence in the results, a number of methodological issues remain outstanding. Specifically, a lack of appropriate comparison/control groups, and unavailability of baseline data leads to an inability to determine if men who choose AS are fundamentally less anxious than those who opt for immediate AT. Although potential predictors of adverse psychological adjustment were identified, these methodological limitations reduce confidence that they fully captured the experience of these men; therefore resulting implications for practice must also be treated cautiously. This lack of confidence is reinforced by conflicting results emanating from 
different research designs. Particularly pertinent were differences in results relating to anxiety and depression between cross-sectional and longitudinal studies. It must be noted that although levels of anxiety and depression appeared to resolve over time when they were followed up longitudinally, attrition and response rate must be considered when evaluating the strength of the evidence. This is reflected in one study [25] that contradicted other longitudinal studies that reported decreasing anxiety and depression at later follow-up points, the same study had one of the lowest rates of attrition with an $84 \%$ response rate. This highlights the importance of reporting reasons for non-response and analysis of potential socio-demographic differences between complete and incomplete responders.

As well as different findings resulting from longitudinal and cross-sectional evidence, further differences were observed in relation to qualitative and quantitative uncertainty data. In terms of prevalence, quantitative studies indicated that uncertainty appears to be low in this population. However when the nature of uncertainty was explored in qualitative studies, it appears to have a more significant impact on men than is reflected in the quantitative data. This idea is comparable to how quality of life is conceptualised in the severity of symptoms versus the 'bother'/impact that is experienced by the patient as a result i.e. the impact of uncertainty on the individual cannot be ignored. Because no qualitative studies reported on anxiety or depression, potential differences in these areas remain moot, requiring further investigation.

\section{Limitations of the included studies}

Some questions need to be raised in relation to the comparators chosen in the reviewed studies. This is a significant issue because it is only through comparison that the extent of difficulties, or perhaps lack thereof, can be fully understood. Several studies included patients opting for AT as comparators, while others compared their results to reference values. Arguably, a more appropriate comparison is age-matched men with no PCa diagnosis in 
addition to patients opting for AT. Due to the high incidence of undiagnosed lower-risk PCa in men over 60 years [36], it can be extrapolated that the psychological differences between patients and age-matched volunteers would be attributable to $\mathrm{PCa}$, and the AS experience. A further criticism of the studies was that, because the patients sampled had already selected AS as a treatment course, it is possible that patients who were naturally less anxious, depressed, or uncertain, chose AS due to increased ability to cope. One study attempted to assess selection bias [21], observed that participants who completed follow-up reported greater psychological dysfunction than those lost to follow-up, illustrating an additional potential bias in terms of the type of patient that remains involved in psychological studies. None of the studies utilised a mixed-methods (MM) design and only a limited number were qualitative, and these only reported results for uncertainty. This is limiting in that men on AS are not being afforded the opportunity to express their interpretation of their experiences. MM research would be of particular benefit in this area, maintaining generalisability while still providing an opportunity to gain a deeper understanding of patients' experiences of AS and PCa generally [7]. The value of this approach is illustrated in men's description of uncertainty. In qualitative studies, participants described overwhelming uncertainty that continued throughout AS [32-34], whereas in quantitative studies, uncertainty reportedly decreased over time. This discrepancy warrants further exploration. This review returned no qualitative papers relating to anxiety or depression; a qualitative study examining these outcomes may have presented different findings, as was the case with uncertainty.

\section{Limitations of the review}

Due to the absence of universal measures for each psychological dimension studied, and indeed consistency in the definitions of each psychological dimension e.g. interchangeable use of the terms distress and depression, it was not possible to combine the data of multiple 
quantitative studies in a meta-analysis. Because of the small number of qualitative studies included, a meta-synthesis was also not feasible.

Although attempts were made to minimise the impact of the use of varying terminology for the process of AS, by using multiple terms in the search strategy (Fig. 1), it remains a possibility that studies using different terminology for AS/studies failing to provide sufficient definitions for the management programme assessed, were not retrieved.

The papers included in this review were also checked against those studies included in previous systematic reviews $[5,6]$; neither review uncovered additional papers, aside from those discussed as limitations previously, i.e. inclusion of WW papers.

\section{Recommendations for future research}

Future research should include appropriate comparison groups, timelier baseline measures, and steps to minimise selection bias. Ideally, baseline assessment should occur prior to treatment decision-making with the aim of assessing patients over time to determine potential temporal variability, thereby controlling for individual differences. The value of longitudinal data has been highlighted, evident in the non-linear declines in anxiety. Although overall anxiety declined up to 30-months, demonstrating increasing resilience over time, anxiety peaked at certain time-points. This fluctuation was particularly pertinent at follow-up appointments or while awaiting results [19]. Cross-sectional data would not have detected this variability.

None of the included studies utilised a MM design. The use of this design would facilitate a more comprehensive understanding of the impact of AS on men, allowing researchers to better understand the holistic needs of patients without compromising generalisability. Finally, researchers should cooperate to standardise the psychological measures used in AS research therefore facilitating comparison and aiding transferability of international AS expertise. 


\section{Recommendations for practice}

Clinicians reading previous reviews and managing the care of men undergoing AS, could easily assume the favourable wellbeing of men receiving AS. Results of the present review discuss the various reasons why this may not necessarily be accurate. Evidence should be interpreted with consideration for the limitations discussed. Patients with favourable-risk PCa deciding on treatment options may require additional reassurance and support when considering AS and continuing this monitoring strategy.

\section{Acknowledgements}

The authors wish to thank Lionne Venderbos, Monique Roobol, Ida Korfage and Lisette ' $t$ Hoen (Erasmus MC Rotterdam) for their support and advice while conducting this systematic review.

\section{References}

1. Lawrentschuk N, Klotz L. Active surveillance for favorable-risk prostate cancer: A short review. Korean Journal of Urology 2010.

2. Klotz L. Active surveillance for prostate cancer: for whom?. Journal of Clinical Oncology 2005;23(32):8165-8169.

3. National Institute for Health and Clinical Excellence. Prostate Cancer: diagnosis and treatment (CG175). 2014. http://www.nice.org.uk/guidance/CG175 (accessed 16 September 2016)

4. Bergman J, Litwin MS. Quality of life in men undergoing active surveillance for localized prostate cancer. Journal of the National Cancer Institute. Monographs. 2012 Sep;2012(45):242.

5. Bellardita L, Valdagni R, van den Bergh R, Randsdorp H, Repetto C, Venderbos LDF, ... Korfage IJ. How Does Active Surveillance for Prostate Cancer Affect Quality of Life? A Systematic Review. European Urology 2014; 1-9. doi:10.1016/j.eururo.2014.10.028 
6. Carter G, Clover K, Britton B, Mitchell AJ, White M, McLeod N, Denham J, Lambert SD. Wellbeing during Active Surveillance for localised prostate cancer: A systematic review of psychological morbidity and quality of life. Cancer Treatment Reviews 2015; 41:46-60.

7. Curry L, Nembhard IM, Bradley EH. Qualitative and mixed methods provide unique contributions to outcomes research. Circulation 2009;119(10):1442-1452. doi:10.1161/CIRCULATIONAHA.107.742775

8. Moher D, Liberati A, Tetzlaff J, Altman DG, The PRISMA Group. Preferred Reporting Items for Systematic Reviews and Meta-Analyses: The PRISMA Statement. BMJ 2009;339:b2535, doi: 10.1136/bmj.b2535

9. Litwin MS, Talcott JA. Measuring quality of life in prostate cancer: progress and challenges. J. Lipscomb, C.C. Gotay, \& C. Snyder (eds). Outcomes Assessment in Cancer . Cambridge, UK: University Press; 2005. pp. 126-159.

10. Pluye P, Robert E, Cargo M, Bartlett G, O’Cathain A, Griffiths F, ... Rousseau MC. Proposal: A mixed methods appraisal tool for systematic mixed studies reviews. Montréal: McGill University; 2011.

11. Pace R, Pluye P, Bartlett G, Macaulay AC, Salsberg J, Jagosh J, Seller R. Testing the reliability and efficiency of the pilot Mixed Methods Appraisal Tool (MMAT) for systematic mixed studies review. International journal of nursing studies 2012; 49(1): 4753.

12. Anderson J, Burney S, Brooker JE, Ricciardelli LA, Fletcher JM, Satasivam P, Frydenberg M. Anxiety in the management of localised prostate cancer by active surveillance. BJU International 2014;114 Suppl: 55-61. doi:10.1111/bju.12765

13. Bellardita L, Rancati T, Alvisi MF, Villani D, Magnani T, Marenghi C, ... Valdagni R. Predictors of Health-related Quality of Life and Adjustment to Prostate Cancer During 
Active Surveillance. European Urology 2013; 64(1): 30-36. doi:10.1016/j.eururo.2013.01.009

14. Burnet KL, Parker C, Dearnaley D, Brewin CR, \& Watson M. Does active surveillance for men with localized prostate cancer carry psychological morbidity? BJU International 2007; 100(3):540-543. doi:10.1111/j.1464-410X.2007.07009.x

15. Couper JW, Love AW, Dunai JV, Duchesne, GM, Bloch S, Costello AJ, Kissane DW. The psychological aftermath of prostate cancer treatment choices: a comparison of depression, anxiety and quality of life outcomes over the 12 months following diagnosis. Medical Journal of Australia 2009;190(7):S86-S89.

16. Goh AC, Kowalkowski MA, Bailey Jr DE, Kazer MW, Knight SJ, Latini DM. Perception of cancer and inconsistency in medical information are associated with decisional conflict: a pilot study of men with prostate cancer who undergo active surveillance. BJU International 2012; 110(2B)

17. Hegarty JM, Wallace M, Comber H. Uncertainty and Quality of Life Among Men Undergoing Active Surveillance for Prostate Cancer in the United States and Ireland. American Journal of Mens Health 2008;2(2):133-142. doi:10.1177/1557988307300467

18. Latini DM, Hart SL, Knight SJ, Cowan JE, Ross PL, DuChane J. The relationship between anxiety and time to treatment for patients with prostate cancer on surveillance. Journal of Urology 2007;178(3):826-831. doi:10.1016/j.juro.2007.05.039

19. Parker PA, Davis JW, Latini DM, Baum G, Wang X, Ward JF, ... Kim J. Relationship between illness uncertainty, anxiety, fear of progression and quality of life in men with favourable-risk prostate cancer undergoing active surveillance. BJU international 2015.

20. Pearce SM, Wang CHE, Victorson DE, Helfand BT, Novakovic KR, Brendler CB, Albaugh JA. A Longitudinal Study of Predictors of Sexual Dysfunction in Men on Active Surveillance for Prostate Cancer. Sexual medicine 2015;3(3):156-164. 
21. Punnen S, Cowan JE, Dunn LB, Shumay DM, Carroll PR, Cooperberg MR. A longitudinal study of anxiety, depression and distress as predictors of sexual and urinary quality of life in men with prostate cancer. BJU International 2013;112(2):E67-75. doi:10.1111/bju.12209

22. Seiler D, Randazzo M, Leupold U, Zeh N, Isbarn H, Chun FK, Kwiatkowski M. Protocolbased Active Surveillance for Low-risk Prostate Cancer: Anxiety Levels in Both Men and Their Partners. Urology 2012;80(3):564-569. doi:10.1016/j.urology.2012.04.053

23. Selli C, Bjartell A, Burgos J, Somerville M, Palacios J-M, Benjamin L, ... Castro, R. Burden of illness in prostate cancer patients with a low-to-moderate risk of progression: A one-year, pan-european observational study. Prostate Cancer 2014; 201:1-8.

24. van den Bergh RCN, Essink-Bot M-L, Roobol MJ, Wolters T, Schröder FH, Bangma CH, Steyerberg EW. Anxiety and distress during active surveillance for early prostate cancer. Cancer 2009;115(17), 3868-78. doi:10.1002/cncr.24446

25. van den Bergh RCN, Essink-Bot M-L, Roobol MJ, Schroder FH, Bangma CH, Steyerberg EW. Do Anxiety and Distress Increase During Active Surveillance for Low Risk Prostate Cancer? Journal of Urology 2010;183(5), 1786-1791. doi:10.1016/j.juro.2009.12.099

26. van den Bergh RCN, Korfage IJ, Roobol MJ, Bangma CH, De Koning HJ, Steyerberg EW, Essink-Bot ML. Sexual function with localized prostate cancer: Active surveillance vs radical therapy. BJU International 2012;110(7), 1032-1039.

27. Venderbos LDF, van den Bergh RCN, Roobol MJ, Schröder FH, Essink-Bot M-L, Bangma $\mathrm{CH}, \ldots$ Korfage IJ. A longitudinal study on the impact of active surveillance for prostate cancer on anxiety and distress levels. Psycho-Oncology 2014; doi:10.1002/pon.3657 
28. Wallace M. Uncertainty and Quality of Life of Older Men Who Undergo Watchful Waiting for Prostate Cancer. Oncology Nursing Forum 2003;30(2), 303-309. doi:10.1188/03.ONF.303-309

29. Watts S, Leydon G, Eyles C, Moore CM, Richardson A, Birch B, ... Lewith G. A quantitative analysis of the prevalence of clinical depression and anxiety in patients with prostate cancer undergoing active surveillance. BMJ open 2015; 5(5), e006674

30. Wilcox CB, Gilbourd D, Louie-Johnsun M. Anxiety and health-related quality of life (HRQL) in patients undergoing active surveillance of prostate cancer in an Australian centre. BJU International 2014;113 Suppl, 64-8. doi:10.1111/bju.12557

31. Yanez B, Bustillo NE, Antoni MH, Lechner SC, Dahn J, Kava B, Penedo FJ. The importance of perceived stress management skills for patients with prostate cancer in active surveillance. Journal of Behavioral Medicine 2014; doi:10.1007/s10865-014-95941

32. Hedestig O, Sandman P-O. Widmark A. Living With Untreated Localized Prostate Cancer. Cancer Nursing 2003; 26(1), 55-60. doi:10.1097/00002820-200302000-00008

33. Kazer MW. Conversion from active surveillance to active treatment for prostate cancer: A qualitative analysis. Journal of Nursing Education and Practice 2012;2(2),80-88. doi:10.5430/jnep.v2n2p80

34. Oliffe JL, Davison BJ, Pickles T, Mroz L. The Self-Management of Uncertainty Among Men Undertaking Active Surveillance for Low-Risk Prostate Cancer. Qualitative Health Research 2009;19(4), 432-443. doi:10.1177/1049732309332692

35. Sharpley CF, Christie DR, Bitsika V. Variability in anxiety and depression over time following diagnosis in patients with prostate cancer. Journal of psychosocial oncology. 2010 Nov 2;28(6):644-65. 
36. Zlotta AR, Egawa S, Pushkar D, Govorov A, Kimura T, Kido M, ... van der Kwast TH. Prevalence of prostate cancer on autopsy: cross-sectional study on unscreened Caucasian and Asian men. Journal of the National Cancer Institute 2013; djt151.

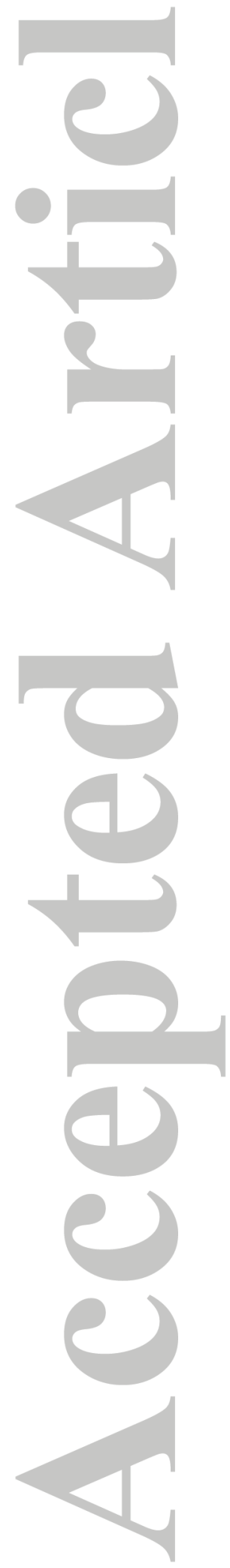




\begin{tabular}{|c|c|c|c|c|c|c|}
\hline Study & Design & $\begin{array}{l}\text { Assessment } \\
\text { period }\end{array}$ & Setting, country; total sample/response rate & $\begin{array}{l}\text { Participants (N; age, years; time on } \mathrm{AS} \text { at } \\
\text { assessment) }\end{array}$ & Comparison & MMAT \\
\hline [12] & Cross-sectional & NR & UH. Australia. 260/33\% & $86 ; 65.7 ; 43$ mths on AS & None & ** \\
\hline [13] & Cross-sectional & $2007-2012$ & CC. Italy. $154 / 67 \%$ & $103 ; 67 ; 10$ months post-biopsy & None & $* * * *$ \\
\hline [14] & Cross-sectional & NR & CC. Britain. 493/67\% & 329,100 AS; $67.12 ; 28.61$ months & $\begin{array}{l}\text { Currently undergoing AT and } \\
\text { Previously underwent AT }\end{array}$ & $* * *$ \\
\hline [15] & Longitudinal & $2001-2005$ & CC. Australia. 211/91.4\% (not split by treatment group) & $\begin{array}{l}\text { T1: } 193-61 \mathrm{WW} ; \mathrm{T} 2: 172-55 \mathrm{WW} ; \\
\text { Demographics not split by treatment group - } \\
66.15 \text { years; Diagnosis/early treatment; } \\
\text { 12months later }\end{array}$ & $\mathrm{RP}, \mathrm{HT}$ & ** \\
\hline [16] & Cross-sectional & $2007-2008$ & Support group database. USA. NR. & $34 ; 63.1 ; 14.1$ months & None & $*$ \\
\hline [17] & Cross-sectional & NR & $\begin{array}{l}\text { Cancer registry, physician referral/advertisement. USA } \\
\text { and Ireland. Irish sample } 92 / 10.8 \% \text {, USA sample NR. }\end{array}$ & $\begin{array}{l}\text { Ireland } 10 ; 76.5 ; \text { mean } 27.6 \text { months } \\
\text { USA } 19 ; 76 ; \text { mean } 48.5 \text { months }\end{array}$ & AS men in south of Ireland and USA & $*$ \\
\hline [18] & Longitudinal & $1997-2002$ & Community/University clinic. USA. NR. & $105 ; 75.5 ;>6$ months & Those who ceased AS for AT & ** \\
\hline [19] & Longitudinal & $2006-2012$ & CC. USA.180/71\% & $180 ; 67.2 ;<6$ months & None & *** \\
\hline [20] & Longitudinal & NR & Database. USA. 195/100\% & 195; 66.5; commencement & None & $* * *$ \\
\hline [21] & Longitudinal & $2007-2010$ & $\begin{array}{l}\text { Urology dept., USA. 864/77\% (Response rate not } \\
\text { divided by treatment group) }\end{array}$ & $122 ; 60.5 ;>6$ months & $\mathrm{RP}$ & $* * * *$ \\
\hline [22] & Cross-sectional & 2010 & CC. Switzerland. 283/44.9\% & $\begin{array}{l}133 \text { couples; patients } 69.3 \text { years; Range } 17-136 \\
\text { months }\end{array}$ & None & ** \\
\hline [23] & Longitudinal & NR & $\begin{array}{l}\text { CC. Germany, France, Spain, Italy, Sweden. 672/48.5\% } \\
\text { (not divided by treatment group) }\end{array}$ & $\begin{array}{l}12 \text { WW/AS; Demographics not split by } \\
\text { treatment type }-65 \text { years; }<2 \text { months of } \\
\text { diagnosis, } 3 \text { months, } 12 \text { months }\end{array}$ & $\begin{array}{l}\text { RP, External beam RT, brachytherapy, } \\
\text { combined HT and RT, HT, RP } \\
\text { followed by salvage RT }\end{array}$ & $* * *$ \\
\hline$[24]^{*}$ & Cross-sectional & $2007-2008$ & UH. The Netherlands. Sample 150/86\%. & $129 ; 64.9 ; 2.2$ months & None & $* * * *$ \\
\hline$[25]^{*}$ & Longitudinal & $2007-2008$ & UH. The Netherlands. $150 / 86 \%$ & $129 ; 64.3$ years; $0-6$ months & None & $* * *$ \\
\hline$[26]^{*}$ & Cross-sectional & NR & UH. The Netherlands. (AS sample only) $150 / 86 \%$ & $129 \mathrm{AS} ; 64.9 ; 6$ and 18 months post-diagnosis & AT & $+* *$ \\
\hline$[27]^{*}$ & Longitudinal & $2007-2008$ & UH. The Netherlands. Sample: 150/86\% & $\begin{array}{l}\text { 129; 64.6; <6months since } \\
\text { diagnosis/commencing AS }\end{array}$ & None & $* * *$ \\
\hline [28] & Cross-sectional & NR & Physician referral/advertisement. USA. NR. & $19 ; 76 ; 4.5$ years & None & * \\
\hline [29] & Cross-sectional & 2012 & CC. England.426/73.47\% & $313 ; 70.49 ;>2$ months, mean NR. & None & * \\
\hline [30] & Cross-sectional & 2013 & Database. Australia. 67/77\% & $47 ; 62 ; \mathrm{NR}$ & None & $* * *$ \\
\hline
\end{tabular}

This article is protected by copyright. All rights reserved. 


\begin{tabular}{|c|c|c|c|c|c|c|}
\hline [31] & Cross-sectional & $2007-2011$ & UC. USA. $452 / 16 \%$ & $71 ; 65.4 ; 16.52$ months & None & ** \\
\hline [32] & $\begin{array}{l}\text { Qualitative, cross- } \\
\text { sectional }\end{array}$ & NR & Database. Sweden. 8/87.5\% & $7 ; 62-6 ; 16-35$ months & None & $* * *$ \\
\hline [33] & $\begin{array}{l}\text { Qualitative, cross- } \\
\text { sectional }\end{array}$ & NR & UC. USA. $6 / 100 \%$ & $6 ; 70 ;>6$ months AS & None & ** \\
\hline [34] & $\begin{array}{l}\text { Qualitative, cross- } \\
\text { sectional }\end{array}$ & NR & CC. Canada. $45 / 55.5 \%$ & $25 ; 68 ;<2$ years & None & $* *$ \\
\hline
\end{tabular}

*The same cohort of 129 participants were studied in multiple papers [25-28]

For further breakdown of the MMAT scores please contact the first author.

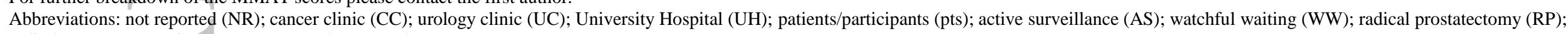
radiotherapy (RT); active treatment (AT); hormone therapy (HT).

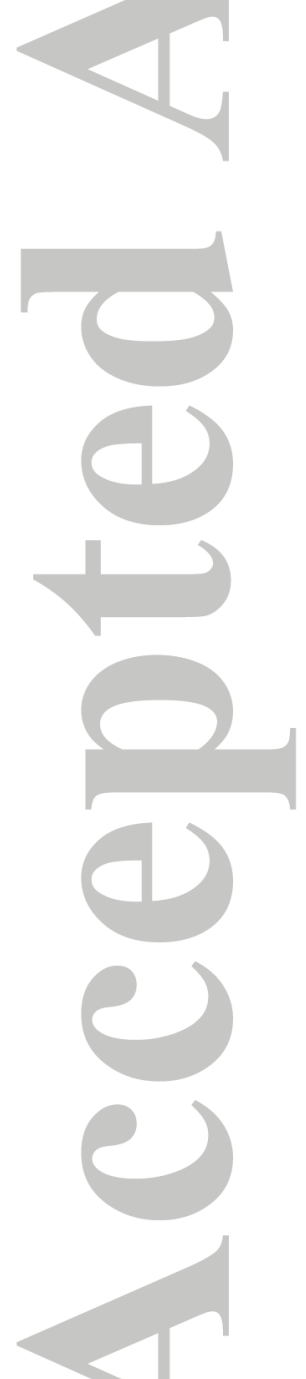


Table 2. Scales used

SCALES

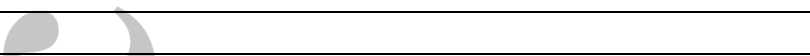

HADS-A: Hospital and Anxiety Depression Scale-Anxiety

subscale

STAI-T: State-Trait Anxiety Inventory-trait subscale

MAX-PC: Memorial Anxiety Scale for Prostate Cancer

Symptom checklist

Mini-MAC: Mini-mental adjustment to cancer

HADS: Hospital and Anxiety Depression Scale

BSI-53: Brief Symptom Inventory

MUIS: Mishel's Uncertainty in Illness Scale

CMS: Fife Constructed Meaning Scale

MHI-5: Mental health index-5

SE: Lepore's self-efficacy for prostate symptom management scale

MUIS-C: Mishel Uncertainty in Illness Scale- Community form

Folkman and Lazarus Appraisal Scale

STAI-S: State-Trait Anxiety Inventory-state subscale

EPIC: Expanded Prostate Cancer Index Composite

SF-12: Short form Health Survey

EPIC-26: Expanded Prostate Cancer Index Composite short form

AUA-SI: American Urological Association - Symptom Index

PHQ-9: Patient health questionnaire

GAD-7: General Anxiety Disorder Scale

DT: Distress thermometer

WPAI: Work Productivity Assessment Index

DCS: Decisional Conflict Scale

CES-D: Centre for Epidemiologic Studies Depression

STAI-6: State-Trait Anxiety Inventory short form

EPQ: Eysenck Personality Questionnaire

Charlson Comorbidities Index

MOCS: Measure of Current Status Scale A

PCPC: The profile of Concerns about PCa

IES-R: Impact of Event Scale-Revised.

Qualitative interviews

*3 items only ** Fear of Recurrence subscale only

\begin{tabular}{|c|c|c|c|c|c|c|c|c|c|c|c|c|c|c|c|c|c|c|c|c|c|c|}
\hline \multicolumn{23}{|c|}{ STUDY } \\
\hline 12 & 13 & 14 & 15 & 16 & 17 & 18 & 19 & 20 & 21 & 22 & 23 & 24 & 25 & 26 & 27 & 28 & 29 & 30 & 31 & 32 & 33 & 34 \\
\hline \multicolumn{23}{|l|}{$\mathbf{X}$} \\
\hline \multicolumn{23}{|l|}{$\mathbf{X}$} \\
\hline $\mathbf{X}$ & & & & $\mathbf{X}$ & & $*$ & $* *$ & $\mathbf{X}$ & & $\mathbf{X}$ & & $\mathbf{X}$ & $\mathbf{X}$ & & $\mathbf{X}$ & $\mathbf{X}$ & & $\mathbf{X}$ & & & & \\
\hline & $\mathbf{X}$ & & & & & & & & & & & & & & & & & & & & & \\
\hline & $\mathbf{X}$ & & & & & & & & & & & & & & & & & & & & & \\
\hline & & $\mathbf{X}$ & & & & & & & & $\mathbf{X}$ & $\mathbf{X}$ & & & & & & $\mathbf{X}$ & & & & & \\
\hline & & & $\mathbf{X}$ & & & & & & & & & & & & & & & & & & & \\
\hline & & & & $\mathbf{X}$ & & & $\mathbf{X}$ & & & & & & & & & & & & & & & \\
\hline & & & & $\mathbf{X}$ & & & & & & & & & & & & & & & & & & \\
\hline & & & & $\mathbf{X}$ & & & & & & & & & & & & & & & & & & \\
\hline & & & & $\mathbf{X}$ & & & & & & & & & & & & & & & & & & \\
\hline & & & & & $\mathbf{X}$ & & & & & & & & & & & $\mathbf{X}$ & & & & & & \\
\hline & & & & & $\mathbf{X}$ & & & & & & & & & & & $\mathbf{X}$ & & & & & & \\
\hline & & & & & & & $\mathbf{X}$ & & & & & & & & & & & & & & & \\
\hline & & & & & & & $\mathbf{X}$ & & & & & & & & & & & & & & & \\
\hline & & & & & & & $\mathbf{X}$ & & & & & & & & & & & & & & & \\
\hline & & & & & & & & $\mathbf{X}$ & & & & & & & & & & & & & & \\
\hline & & & & & & & & $\mathbf{X}$ & & & & & & & & & & & & & & \\
\hline & & & & & & & & & $\mathbf{X}$ & & & & & & & & & & & & & \\
\hline & & & & & & & & & $\mathbf{X}$ & & & & & & & & & & & & & \\
\hline & & & & & & & & & $\mathbf{X}$ & & & & & & & & & & & & & \\
\hline & & & & & & & & & & & $\mathbf{X}$ & & & & & & & & & & & \\
\hline & & & & & & & & & & & & $\mathbf{X}$ & $\mathbf{X}$ & & $\mathbf{X}$ & & & & & & & \\
\hline & & & & & & & & & & & & $\mathbf{X}$ & $\mathbf{X}$ & $\mathbf{X}$ & $\mathbf{X}$ & & & & & & & \\
\hline & & & & & & & & & & & & $\mathbf{X}$ & $\mathbf{X}$ & $\mathbf{X}$ & $\mathbf{X}$ & & & & & & & \\
\hline & & & & & & & & & & & & $\mathbf{X}$ & $\mathbf{X}$ & & & & & & & & & \\
\hline & & & & & & & & & & & & & & & & & & & $\mathbf{X}$ & & & \\
\hline & & & & & & & & & & & & & & & & & & & $\mathbf{X}$ & & & \\
\hline & & & & & & & & & & & & & & & & & & & $\mathbf{X}$ & & & \\
\hline & & & & & & & & & & & & & & & & & & & $\mathbf{X}$ & & & \\
\hline & & & & & & & & & & & & & & & & & & & & $\mathbf{X}$ & $\mathbf{X}$ & $\mathbf{X}$ \\
\hline
\end{tabular}

This article is protected by copyright. All rights reserved. 


\begin{tabular}{|c|c|c|}
\hline & Psychological outcome & \\
\hline$\uparrow$ Anxiety & $\uparrow$ Depression & $\uparrow$ Uncertainty \\
\hline $\begin{array}{l}\downarrow \text { Number of cores at biopsy [12] } \\
\downarrow \text { Age [13, 24] } \\
\uparrow \text { Time since diagnosis [13] } \\
\text { Receipt of curative treatment [17] } \\
\downarrow \text { QoL [18] } \\
\uparrow \text { Fear of progression [18] } \\
\downarrow \text { Time on AS [12, 18, 19, 24] } \\
\uparrow \text { Role of the physician }[12,23, \\
29] \\
\downarrow \text { QoL [23] } \\
\text { MH impairment [12] } \\
\uparrow \text { Neuroticism [23] } \\
\uparrow P S A[23] \\
\uparrow \text { Uncertainty [27] } \\
\text { Divorce, lack of partner [12, 28] } \\
\downarrow \text { PCa knowledge [29] } \\
\downarrow \text { Coping [30] } \\
\uparrow P C a \text { concerns [30] } \\
\downarrow \text { Optimism [30] }\end{array}$ & $\begin{array}{l}\uparrow \text { Time since diagnosis [13] } \\
\downarrow \text { QoL [13] } \\
\uparrow \text { Neuroticism [24] }\end{array}$ & $\begin{array}{l}\downarrow \text { Time since diagnosis [18] } \\
\downarrow \text { QoL [18] } \\
\uparrow \text { Fear of progression [18] } \\
\uparrow \text { Role of the physician } \uparrow[24] \\
\uparrow \text { Neuroticism [24] } \\
\uparrow \text { Anxiety [27] } \\
\uparrow \text { Perception of danger [27] } \\
\text { Perceptions of being 'alone' [31] } \\
\text { Compromised masculinity [31, 33] } \\
\text { Conflicting relationship with physician } \\
\text { [31, 32] } \\
\text { Fear/worry, 'risking one’s life' [31, } \\
32] \\
\text { Fear of treatment failure/disease } \\
\text { recurrence/spread [32, 33] } \\
\uparrow \text { Side effects [32, 33] } \\
\text { Temporal variability of uncertainty } \\
\text { [33] } \\
\downarrow \text { age and increased biopsy pain - } \\
\text { patients compared biopsy with rape } \\
\text { [33] }\end{array}$ \\
\hline
\end{tabular}




\section{Figure 1. PRISMA Flow Diagram (study selection and search terms}

Search terms

Prostatic Neoplasms [MeSH] OR Prostat* AND (Cancer OR Tumour OR Tumor OR Neoplasm) AND (Active Surveillance OR Disease Surveillance OR Expectant Treatment OR Expectant Management OR Watchful Waiting OR Observation) AND (Stress, Psychological [MeSH] OR Anxiety [MeSH] OR Quality Of Life [MeSH] OR Uncertainty [MeSH] OR Psychology [MeSH] OR Adaptation, Psychological [MeSH] OR Psych* OR Coping).

Records identified through database searching (CINAHL, InterNurse, Embase, Medline, PsycINFO, PsycARTICLES, and Web of Science). $(n=11270)$

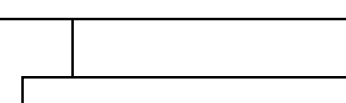

Records after duplicates removed $(n=9412)$

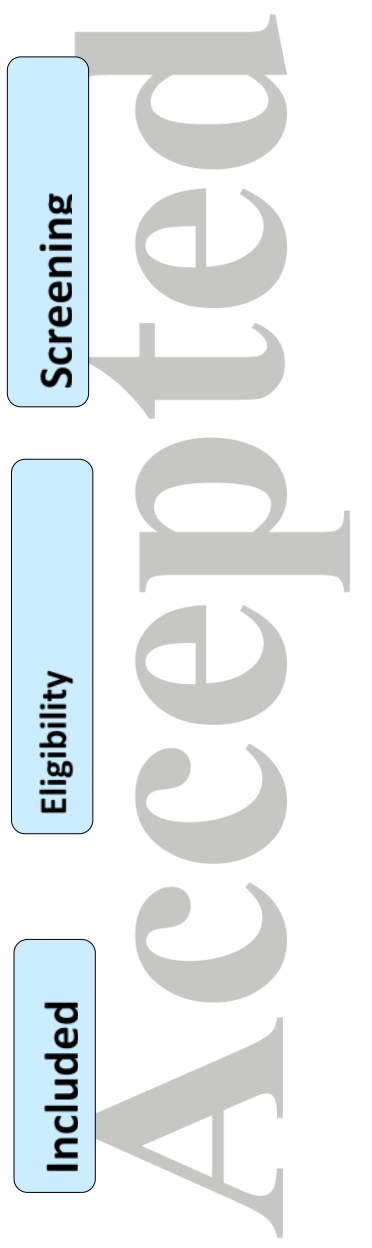

Additional records identified through other sources (reference lists and Google Scholar

$$
(n=10)
$$

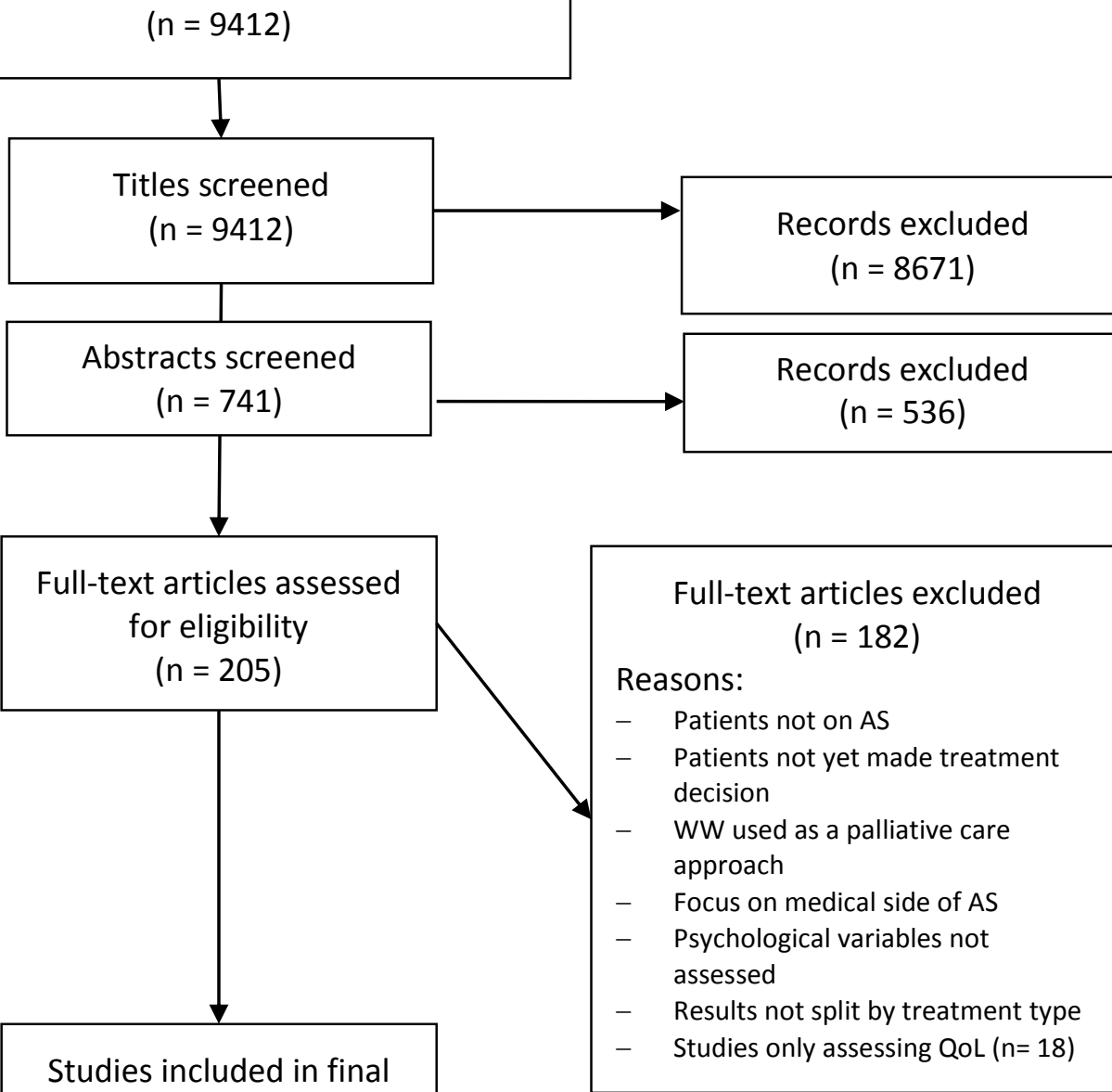

This article is protected by copyright. All rights reserved. 